\title{
Progressive chorea and dystonia associated with a large arteriovenous malformation
}

\author{
Jeffrey S Raskin ${ }^{1}$, Mered Parnes ${ }^{2}$, Sandi Lam ${ }^{3}$ \\ ${ }^{1}$ Section of Pediatric Neurosurgery, Riley Hospital for Children, Department of Neurological Surgery, \\ Indiana University School of Medicine, Goodman Campbell Brain and Spine, Indianapolis, Indiana, \\ ${ }^{2}$ Pediatric Movement Disorders Clinic, Section of Pediatric Neurology and Developmental \\ Neuroscience, Baylor College of Medicine/Texas Children's Hospital, Houston, Texas, ${ }^{3}$ Department of \\ Neurosurgery, Division of Pediatric Neurosurgery, Baylor College of Medicine/ Texas Children's \\ Hospital
}

Corresponding author: Sandi Lam; Email: sklam@texaschildrens.org

doi) https://doi.org/10.17724/jicna.2019.154

Received: 14 June 2018

Accepted: 14 October 2019

\begin{abstract}
A 9-year-old male with intellectual disability and epilepsy presenting with a progressive movement disorder characterized by chorea and dystonia primarily affecting the left face, arm, and leg and attributed to an arteriovenous malformation centered within the right thalamus with bilateral extension into the basal ganglia, midbrain and pons is reported.
\end{abstract}

Kewords: dystonia,basal ganglia,arteriovenous malformation.

(C) Raskin JS; licensee JICNA

\section{Case Report}

A nine-year-old male with intellectual disability and epilepsy presented with a progressive movement disorder characterised by chorea and dystonia primarily affecting the left face, arm, and leg.

Neuroimaging revealed a diffuse Spetzler-Martin Grade IV arteriovenous malformation centered within the right thalamus with bilateral extension into the basal ganglia, midbrain and pons. An associated network of developmental venous anomalies was noted to coalesce into ectatic deep drainage, causing obstructive hydrocephalus (Figure 1). Surgical management was not indicated given the anatomy and location of the lesion.

The patient presented with a stepwise clinical decline over several months, with worsening chorea and dystonia resulting in loss of function of the left arm, severe left arm pain, and loss of the ability to ambulate independently (See supplementary file at http://icnapedia.org/s/148). The involuntary movements and arm pain improved following treatment with clonazepam, baclofen, tetrabenazine, and hydrocodone/acetaminophen as well as chemodenervation with intramuscular botulinum toxin. The patient developed somnolence in the setting of worsening hydrocephalus. Following the ventriculoperitoneal shunt, he experienced a

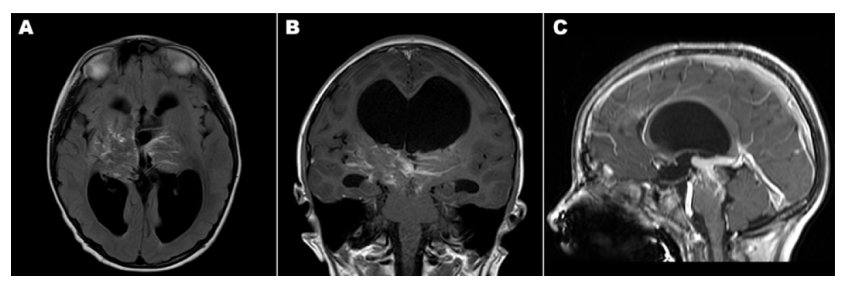

Figure 1 A) Axial B) coronal and C) sagittal T1 MRI with gadolinium demonstrating extensive, bilateral arteriovenous malformation within basal ganglia, thalami and midbrain, and obstructive hydrocephalus.

dramatic improvement in his level of awareness, without further improvement in his involuntary movements.

\section{Discussion \& Conclusion}

The pathogenesis of secondary movement disorders is varied; etiologies include hypoxia, stroke, and kernicterus [1]. Movement disorders resulting from congenital vascular malformations are relatively uncommon and can be difficult to treat. Apart from producing structural abnormalities, which can physically disrupt normal basal ganglia-thalamocortical 
circuits, vascular malformations can further complicate regional dysfunction by vascular steal phenomena [2]. In this case, surgical and medical management has been helpful. Intrathecal baclofen therapy may be an option in the future should symptoms become refractory to medical interventions.

Appropriate consent was obtained from the family for video use within this publication.

\section{Competing interests}

The authors have declared that they have no competing interests.

\section{Author contributions}

All the authors contributed to data collection and also critically reviewed the manuscript. The final version of the manuscript was approved by all the authors.

This is an Open Access article distributed under the terms of the Creative Commons Attribution License (http://creativecommons.org/licenses/by/4.0), which permits unrestricted use, distribution, and reproduction in any medium, provided the original work is properly credited. The Creative Commons Public Domain Dedication waiver (http://creativecommons.org/publicdomain/zero/1.0/) applies to the data made available in this article, unless otherwise stated.

Cite this article as:

Raskin JS, Parnes M, Lam S. Progressive chorea and dystonia associated with a large arteriovenous malformation. JICNA [Internet]. 2019Oct.4. Available from: https://jicna.org/index.php/journal/article/view/jicna-

2019-148

\section{References}

[1] Netravathi M, Pal PK, Indira Devi B. A clinical profile of 103 patients with secondary movement disorders: correlation of etiology with phenomenology. Eur J Neurol. 2012 Feb;19(2):226-233. PubMed.

[2] Park J. Movement Disorders Following Cerebrovascular Lesion in the Basal Ganglia Circuit. J Mov Disord. 2016 May;9(2):71-79. PubMed. 